\title{
I posti dei rifiuti. Badara Ngom e la discarica di Mbeubeuss
}

A Place for Waste. Badara Ngom and Mbeubeuss

\section{Luca Rimoldi}

\section{OpenEdition}

1 Journals

\section{Edizione digitale}

URL: http://journals.openedition.org/aam/3348

DOI: $10.4000 /$ aam.3348

ISSN: 2038-3215

Editore

Dipartimento Culture e Società - Università di Palermo

Notizia bibliografica digitale

Luca Rimoldi, «l posti dei rifiuti. Badara Ngom e la discarica di Mbeubeuss», Archivio antropologico mediterraneo [Online], Anno XXIII, n. 22 (2) | 2020, online dal 31 décembre 2020, consultato il 28 janvier 2021. URL: http://journals.openedition.org/aam/3348 ; DOI: https://doi.org/10.4000/aam.3348

Questo documento è stato generato automaticamente il 28 janvier 2021.

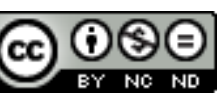

Archivio antropologico mediterraneo è distribuita con Licenza Creative Commons Attribuzione - Non commerciale - Non opere derivate 4.0 Internazionale. 


\title{
I posti dei rifiuti. Badara Ngom e la discarica di Mbeubeuss
}

\author{
A Place for Waste. Badara Ngom and Mbeubeuss
}

\author{
Luca Rimoldi
}

\section{Introduzione}

1 Questo contributo descrive alcuni tratti della vita sociale dei rifiuti, inserendosi nel dibattito sull'antropologia dei rifiuti (Alliegro 2017, 2018; Drackner 2005; Reno 2014; Fredericks 2018; Eriksen 2017, Alexander, O'Hare 2020). Dopo aver preso in considerazione la categoria di rifiuto, descriverà gli spazi di vita e di lavoro della discarica di Mbeubeuss ${ }^{1}$, nella periferia di Dakar. I rifiuti sono in grado di creare relazioni e spazi, delimitati e definiti dalle politiche e dalle pratiche (formali e informali) della loro stessa gestione (Thompson 2017). La letteratura storico-sociologica relativa ai paesi occidentali contemporanei mostra come gli immaginari relativi ai rifiuti non siano più strettamente collegati a questioni che riguardano la salute del corpo individuale (Rathje, Murphy 1992, O'Brien 2008), ma sembrano essere connessi a questioni relative al corpo sociale ed espressi nei termini di rischio ambientale (Melosi 2005). Come scrive l'antropologo Joshua Reno ${ }^{2}$ : «Ritratti come lo sporco segreto che deve essere rivelato, i rifiuti nordamericani sono spesso utilizzati per risvegliare preoccupazione sullo sfruttamento delle risorse da parte degli esseri umani e sull'inquinamento del pianeta nella sua totalità» (Reno 2016: 2). In questo senso un'antropologia dei rifiuti dovrebbe essere in grado di riconnettere i cittadini con le discariche per mettere in evidenza le relazioni esistenti tra i produttori di rifiuti e i luoghi deputati alla loro gestione. L'analisi della gestione delle fasi di raccolta, trasporto ed eliminazione dei rifiuti getta luce anche sulla prossimità fisica e categoriale in cui si trovano a vivere diversi gruppi di persone, specialmente ai margini delle città contemporanee (Bauman 2007, Fredericks 2018, Millar 2018). In linea generale, come sottolinea Enzo Alliegro (2018), la gestione dei rifiuti: 
è presa in carico da personale autorizzato che la immette in un circuito fatto di mezzi di trasporto, siti di stoccaggio, centri di trasferenza, per prendere poi la strada del trattamento che si basa su impianti di selezione, tritovagliatura, combustione, compostaggio, sino ad arrivare all'inevitabile conferimento delle frazioni residue in discarica. Attraverso fasi sempre più laboriose che la procedura tecnica prevede, i rifiuti seguono questo itinerario che li conduce a farsi attanti pubblici. Ed è proprio questa complessa filiera multisituata a generare molteplici possibilità di studio di taglio antropologico (Alliegro 2018: 142).

2 Da questo punto di vista, l'osservazione delle pratiche legate ai rifiuti - dalla loro «produzione» alla loro eventuale distruzione o al recupero e riutilizzo - è in grado di rivelare i campi di forza che regolano la vita sociale e culturale delle società o, come sostiene Guido Viale: «i rifiuti sono un documento diretto, minuzioso e incontrovertibile delle abitudini e dei comportamenti di chi li ha prodotti, anche al di là delle sue stesse convinzioni o della percezione che ha di se stesso» (Viale 1994: 28-29).

Pertanto, i rifiuti rappresentano una sfida per le scienze sociali e devono essere considerati come oggetti di ricerca particolarmente densi - nella loro materialità - per quelle discipline che, come l'antropologia, sono interessate allo studio degli aspetti solitamente meno indagati $\mathrm{e}$, di riflesso, più profondamente incorporati nelle vite delle persone. Come sostiene Reno, infatti, scopo dell'antropologia è quello di studiare l'eliminazione dei rifiuti come una relazione sociale e non, semplicemente, come un abuso sull'ambiente. È proprio in questo senso che si può leggere uno dei racconti di Italo Calvino, La poubelle agréée, in cui l'autore descrive accuratamente le possibili relazioni sociali che stanno dietro al gesto quotidiano del portare fuori l'immondizia. Tali relazioni costituiscono parte di un waste regime (Reno 2016: 2) che include consumatori e produttori e che implica la condizione necessaria per rendere possibile un certo stile di vita - «The garbage keeps coming», come disse Bob, direttore delle operazioni alla discarica di Four Corners, all'antropologo. Ritengo pertanto necessario esplicitare i significati che la categoria di rifiuti assume, da una parte, e le pratiche di lavoro ad essi legati, dall'altra, attraverso l'etnografia condotta presso la discarica di Mbeubeuss.

Questo articolo intende dimostrare come il valore non sia solamente una rappresentazione delle relazioni sociali, ma come esso contribuisca a mantenere sistemi di potere e gerarchie legati ai rifiuti (Thompson 1979). Pertanto, propongo una triplice lente di analisi per indagare gli scarti. Da una parte prendo in considerazione i rifiuti come categoria teorica e metto in luce come alla base del pensiero occidentale i rifiuti siano percepiti come qualcosa che sfugge a una rigida categorizzazione. Attraverso la letteratura e, in particolar modo, applicando uno sguardo etnografico al racconto di Italo Calvino e a un dialogo di Paul Valéry, intendo sottolineare come, nella realtà urbana, ciò che sfugge alle categorie analitiche è invece in grado di diventare simbolo delle relazioni tra individui e società. Da ultimo, attraverso la descrizione di alcuni dettagli etnografici da me raccolti presso la discarica di Mbeubeuss, metto in luce la relazione tra spazio e rifiuti attraverso alcuni episodi della vita di Badara Ngom, un recuperatore della discarica di Dakar ${ }^{3}$. Attraverso l'intreccio di questi approcci sono convinto di poter mostrare la complessità degli oggetti di scarto, visti nella loro dimensione spaziale. Inoltre, il caso della discarica di Mbeubeuss risulta particolarmente significativo dal momento che qui, come in molte altre realtà del sud del mondo, alle figure di "consumatori" e "produttori" individuate da Reno è possibile 
aggiungere quella dei "recuperatori", che rimettono in circolazione rifiuti recuperati andando così ad ampliare quello che Reno ha definito «regime dei rifiuti».

\section{Oggetti e rifiuti}

5 Nel racconto La poubelle agréée $e^{4}$, Italo Calvino mette in luce la relazione tra la sfera domestica e quella pubblica descrivendo in termini che si potrebbero definire per certi aspetti auto-etnografici la sua relazione con la pratica quotidiana del gettare la spazzatura 5 .

Delle faccende domestiche, l'unica che io disimpegni con qualche competenza e soddisfazione è quella di mettere fuori l'immondizia. L'operazione si divide in varie fasi: prelievo della pattumiera di cucina e suo svuotamento nel recipiente più grande che sta nel garage, poi trasporto del detto recipiente sul marciapiede fuori della porta di casa, dove verrà raccolto dagli spazzini e vuotato a sua volta nel loro autocarro (Calvino 1994: 59).

6 Una palazzina unifamiliare nella Parigi degli anni Settanta fa da sfondo al rito laico quotidiano che consente allo scrittore di eliminare gli scarti attraverso azioni minime del singolo - «mi costituisco primo ingranaggio d'una catena d'operazioni decisive per la convivenza collettiva, sancisco la mia dipendenza dalle istituzioni senza le quali morirei sepolto dai miei stessi rifiuti nel mio guscio di individuo singolo» (Calvino 1994: 62) - guidate da un agreement con la città, la sua regolamentazione e i suoi rappresentanti $i^{6}$. Tuttavia, gli scarti, o meglio, ciò che viene ritenuto materiale di scarto, cambia in base al contesto geografico, storico, politico e sociale e altrettanto variabile è la loro gestione. Gli studi antropologici e sociologici sui rifiuti hanno messo in luce la tendenza, apparentemente universale, a classificare alcune cose - così come alcune persone - come inquinate e come tali classificazioni rispondano ad esigenze umane di ricerca del senso e del significato. Tale aspetto non sfugge allo sguardo di Italo Calvino, che sottolinea la necessità della presenza di intermediari in grado di far rispettare l'accordo tra il singolo e la collettività, tra il cittadino e la città e, contemporaneamente, di far procedere il rito di purificazione. Gli éboueurs (letteralmente: sfangatori) della città di Parigi degli anni Settanta sono quasi sempre originari della «maldecolonizzata Africa» (Ibidem: 69) e svolgono una professione precaria "compiuta da chi non è ancora riuscito a mettere radici nella metropoli» (Ibidem: 68) che, tuttavia, rappresenta l'ingresso nel mondo dei consumi attraverso la gestione della fase finale del processo industriale di produzione e distribuzione delle merci. È lo stesso Calvino che, ne «Le città invisibili» (1972), descrive la relazione tra gli abitanti della città di Leonia e i rifiuti da loro stessi prodotti sottolineando, ancora una volta, la relazione tra consumo e affermazione di uno status identitario. Il paesaggio di Leonia, città che «rifà se stessa ogni giorno», viene descritto con queste parole:

Sui marciapiedi, avviluppati in tersi sacchi di plastica, i resti di Leonia d'ieri aspettano il carro dello spazzaturaio. Non solo i tubi di dentifricio schiacciati, lampadine fulminate, giornali, contenitori, materiali d'imballaggio, ma anche scaldabagni, enciclopedie, pianoforti, servizi di porcellana: più che dalle cose che ogni giorno vengono fabbricate vendute comprate, l'opulenza di Leonia si misura dalle cose che ogni giorno vengono buttate via per far posto alle nuove (Calvino 2016: 111-112).

7 Dove i rifiuti vengano ogni giorno trasportati non è di alcun interesse per gli abitanti della città, fintanto che questi scompaiano dalla loro vista, «ma ogni anno la città s'espande, e gli immondezzai devono arretrare più lontano; l'imponenza del gettito 
aumenta e le cataste s'innalzano, si stratificano, si dispiegano su un perimetro più vasto» (Calvino 2016:112).

Nell'Eupalinos o l'Architetto di Paul Valéry (1989), l'ombra di Socrate e quella di Fedro dialogano nell'Ade. Socrate racconta a Fedro che, durante l'adolescenza, furono l'incontro causale con un oggetto e le riflessioni suscitate da tale incontro ad averlo instradato alla filosofia. Stupito dalla rivelazione di Socrate, Fedro inizia a interrogarlo sulla natura di tale ritrovamento. Socrate riferisce che, durante una passeggiata in riva al mare, trovò per caso un oggetto dalla natura ambigua, che, probabilmente, era stato rigettato dal mare.

Socrate: [...] Fu il caso a mettermi in mano l'oggetto più ambiguo del mondo [...] Ebbene, Fedro, ecco come andò: camminavo sulla riva del mare, seguivo una spiaggia senza fine... Non ti sto raccontando un sogno. Andavo non so dove, troppo pieno di vita, mezzo ebbro della mia giovinezza [...]. ogni cosa intorno a me era semplice e pura: il cielo, la sabbia, l'acqua [...].

Fedro: $\mathrm{E}$ l'oggetto?

Socrate: Giace sulla riva dove camminavo, dove mi sono fermato [...]. Ho trovato una di quelle cose rigettate dal mare, una cosa bianca e della più pura bianchezza. Levigata, dura, dolce, leggera, brillava al sole sulla sabbia appianata, oscura e cosparsa di scintille. L'ho presa, vi ho soffiato sopra, l'ho strofinata sul mio mantello, e la sua forma singolare ha fermato ogni mio pensiero. Chi ti ha fatto? Pensai. Tu non assomigli a niente, eppure non sei informe. Sei tu il gioco della natura, o cosa senza nome, a me giunta per invio degli dèi tra le immondizie ripudiate stanotte dal mare? [...] Rimasi un poco e per metà del tempo a considerarla in tutte le sue facce. La interrogai senza aspettarmi risposta... che quel singolare oggetto fosse opera della vita, dell'arte o anche del tempo e gioco della natura? Non potevo discernere... Allora, improvvisamente, lo rigettai in mare. [...] Supponi dunque ch'io consideri una cosa molto familiare come una casa, un tavolo, un'anfora, e che io finga per qualche tempo di essere un uomo selvaggio e di non aver mai visto oggetti simili: avrei ragione di dubitare che questi oggetti siano di fabbricazione umana... Non sapendo a cosa possano servire e, anzi, nemmeno se riescano ad essere in qualche modo utili a qualcuno, e non essendovi, peraltro, chi possa informarmi, bisogna che io escogiti il modo di acquietare il mio spirito a tal proposito... (Valéry 2011: 45-50).

La natura dell'oggetto che Socrate trova sulla spiaggia «richiesto o ripudiato parimenti da tutte le categorie» (Valéry 2011: 50) sfugge alla sua comprensione - il filosofo non può fare a meno di gettarlo via, rifiutandolo (cioè rendendolo un rifiuto). Tuttavia, l'oggetto stimola la sua curiosità al punto tale da porsi domande sulla sua funzione e sulla sua origine (umana o divina). Il gesto di rigettarlo in mare, che a un primo livello di analisi potrebbe apparire come una negazione della sua stessa "scoperta», in realtà si rivela essere la scintilla che contribuisce a dare forma a un'idea. Come detto, nel dialogo che Socrate e Fedro intrattengono l'oggetto che suscita la curiosità di Socrate viene raccolto «tra le immondizie ripudiate [...] dal mare [...]» (Valéry 2011: 47). Per converso, il giovane Socrate di Platone, impegnato in un dialogo con Parmenide a proposito della dottrina delle idee, non sembra mostrare la stessa attenzione agli scarti. Se nell'Ade, il problema di Socrate sembra essere paragonabile al problema dei rifiuti (Thill 2015), nel Parmenide questi appaiono «fuori posto» anche nell'iperuranio. Come è noto, secondo la dottrina delle idee, la conoscenza di qualcosa è possibile solamente a condizione di conoscere il modello, l'idea di questo qualcosa; le idee, dal canto loro, possiedono uno statuto di esistenza indipendente da ciò di cui rappresentano il modello e, nella riflessione platonica, sono collocate in ciò che Socrate chiama iperuranio. La questione dei rifiuti sembra mettere in difficoltà anche il Socrate platonico: interrogato 
dal filosofo di Elea sulla possibilità che nell'iperuranio dimorino anche le idee di realtà «che sembrano ridicole, come capelli, fango, sporcizia [’ं́́noc] e altro privo di importanza e valore» (Platone 130C: 381$)^{7}$, Socrate risponde stizzito negando con forza ai rifiuti la possibilità di esistenza in termini di idee nell'iperuranio platonico - «No! ribatté Socrate -. Io credo invece che quelle cose che vediamo esistano così come le vediamo, mentre mi sembra un po' assurdo credere che vi sia qualche idea di queste» (Platone 130D: 381). Di fronte a una negazione così netta, Parmenide non può fare altro che accusare la giovane età di Socrate e la conseguente inesperienza (cfr. Migliori 2000: 467, nota 11): «Certo, Socrate, sei ancora giovane - disse Parmenide - e la filosofia non ti ha ancora preso come, a mio avviso, ti prenderà il giorno in cui non disprezzerai più nessuna di queste realtà. Ora, invece, a causa della tua età, tieni in considerazione le opinioni degli uomini» (Platone 130E: 381).

Se la categoria di rifiuto o quella di scarto può accomunare, a un primo livello di analisi, numerose realtà sociali non bisogna dimenticare che, come sostiene Thompson: «Su basi culturali le persone possono attribuire valore a cose diverse, oppure possono attribuire un diverso valore alla stessa cosa, ma tutte le culture insistono su alcune distinzioni tra ciò che ha valore e ciò che non ne ha» (Thompson 2017: 20). In lingua inglese i termini garbage, trash, refuse, rubbish, discard sono spesso utilizzati come sinonimi, benché abbiano significati differenti. Se trash fa riferimento a quei rifiuti teoricamente secchi (rifiuti inorganici) e se garbage a quelli umidi (rifiuti organici), il termine refuse include entrambe le tipologie di scarti, così come rubbish, che, tuttavia si riferisce anche agli scarti dei cantieri. Il termine waste di origine latina (vastus, deserto, vuoto, saccheggiato, distrutto) è principalmente riferito a terre o regioni, indica lo stato di abbandono di qualcosa, mentre discard si riferisce all'azione più che al suo oggetto, indicando letteralmente il gettare via una carta da gioco. In lingua francese il sostantivo maschile déchet, derivato di déchoir, trova origine nel termine latino cadere preceduto dal prefisso dis-, che indica separazione o distanza; ordures, dal francese antico ordu, deriva dal latino horridus e sta a indicare tutto ciò che suscita una sensazione fisica che fa accapponare la pelle e rizzare i peli o i capelli, cioè ciò che è disordinato e sporco. In lingua italiana, tra i sinonimi di rifiuto, si trovano immondizia, pattume, sporcizia, ciarpame, spazzatura. Se pattume (dal latino pactus, compatto) e ciarpame (da ciarpa - sciarpa - e, per estensione, insieme di cose considerate inutili) fanno riferimento alla materialità degli oggetti in questione, sporcizia e immondizia fanno riferimento a loro caratteristiche acquisite. Più interessante, a mio avviso, il termine spazzatura: esso infatti indica sia l'azione di spazzare sia l'insieme dei rifiuti e deriva dal verbo latino spatiari (da spatium), inteso nel significato di «fare spazio». Come vedremo, la questione dello spazio risulta centrale nell'analisi della vita sociale dei rifiuti, basti pensare che la stessa Mary Douglas (1966), identifica come sporco tutto ciò che in una società è considerato "fuori posto» (out of place). ${ }^{8}$ Tuttavia, il caso di Mbeubeuss, dei lavoratori e delle lavoratrici della discarica sembra suggerire che questi ultimi costruiscano spazi e tempi di lavoro e socialità che, appunto, si strutturano attorno alla presenza e alle pratiche di gestione dei rifiuti stessi.

\section{Il campo dei rifiuti e Badara Ngom}

11 Mbeubeuss è il nome della discarica di Dakar, la più estesa dell'Africa dell'ovest che occupa lo spazio (86 ettari circa, in continua espansione) di Mbeubuesse, un lago salato 
prosciugato. Inaugurata sul finire degli anni Sessanta come luogo di deposito dei rifiuti solidi urbani, dista circa trenta chilometri dal centro della capitale senegalese. Attualmente lo spazio della discarica appare incapsulato tra le abitazioni di alcuni quartieri dei comuni di Malika e Keur Massar - istituiti nella seconda metà degli anni Novanta come parti dell'arrondissement di Pikine. Le geografie sociali ed economiche di Mbeubeuss cambiano costantemente seguendo sia la scansione del tempo della giornata lavorativa, sia i passaggi dalla stagione secca a quella delle piogge, sia il verificarsi di eventi catastrofici più circostanziali (come gli incendi e gli allagamenti). La prolungata presenza sul campo mi ha permesso di cogliere l'estrema dinamicità del contesto, che, tuttavia, si costruisce anche tramite eventi ripetitivi su base giornaliera, come l'arrivo di centinaia di camion che versano tonnellate di rifiuti solidi indifferenziati in specifiche aree della discarica9. Tali rifiuti sono raccolti dai boudioumane, i recuperatori e le recuperatrici che lavorano nelle piattaforme di versamento, e rivenduti attraverso specifiche reti relazionali ed economiche. Ciò rende evidente il fatto che la discarica non sia solamente luogo per rifiuti. Mbeubeuss è una discarica «selvaggia»: l'assenza di mura perimetrali consente a chiunque di accedere - a piedi - a qualunque luogo senza passare dall'ingresso principale, da cui, solitamente, entrano anche i camion. Come detto, centinaia di persone vivono e lavorano al suo interno: nel tempo, sono stati costruiti due insediamenti stabili nell'area della discarica, che hanno dato vita a tre macro-gruppi di lavoratori informali: quelli stanziati a Gouye-gui, quelli stanziati a Baol (detti Baol-Baol) e quelli stanziati nel percorso che collega l'ingresso della discarica a Gouye-gui (detti Abord) ${ }^{10}$. Sebbene tutte le persone che lavorano in discarica abbiano a che fare con i rifiuti (non foss'altro per il contatto fisico diretto), non tutti sono recuperatori (boudioumane). La stratificazione e il tessuto - sociale ed economico costruitisi a partire dalla fine degli anni Sessanta sono articolati e disomogenei. Ai recuperatori si affiancano diverse figure professionali del mercato informale: i boroompàkk (grossisti e semi-grossisti che acquistano e rivedono materie recuperate all'interno e all'esterno della discarica), i boroom-charette (trasportatori), i venditori e le venditrici, le ristoratrici - ma anche gli agricoltori e gli allevatori di vacche e di maiali - sono solamente alcuni esempi. A mio avviso, dunque, è la presenza stessa dei rifiuti nella discarica, a un primo livello di analisi, ciò che crea relazioni di tipo economico tra le varie figure del lavoro informale in discarica: molto spesso, infatti, un recuperatore "si specializza" nel recupero di un certo tipo di materiale e la vendita di quest'ultimo lo porterà a intessere relazioni con semi-grossisti e grossisti interessati al suo acquisto $\mathrm{e}$ alla sua successiva vendita. Allo stesso modo, un recuperatore tende ad acquistare il cibo e l'acqua sempre dalla stessa ristoratrice dal momento che, grazie alla costruzione di una relazione di fiducia prolungata nel tempo, è più probabile che cibo e acqua vengano concessi a credito, quando necessario.

12 I rifiuti creano anche spazi: lo spazio della piattaforma di sversamento - luogo in cui i recuperatori e le recuperatrici trascorrono la maggior parte della giornata lavorativa aspettando i camion e smistando i rifiuti della giornata - gli spazi dei pàkk - gli insediamenti dei grossisti dove i recuperatori vendono ciò che hanno raccolto - le strade di collegamento tra questi luoghi - vie percorse su carretti trainati da cavalli e guidati da boroom-charrette ${ }^{11}$ - gli spazi attorno ai punti di ristoro - dove molte donne cucinano e vendono cibo, acqua e café touba ai recuperatori in pausa. Nonostante ci siano importanti differenze, la percezione dello spazio della discarica da parte dei miei interlocutori e delle mie interlocutrici è intimamente collegata alla possibilità di poter svolgere un lavoro che permetta loro di costruirsi una vita e di sostentare il proprio 
gruppo famigliare. Se i rifiuti sono fuori posto, il posto dei rifiuti risulta cruciale nelle traiettorie di vita e di lavoro dei lavoratori informali della discarica. Sebbene molte delle storie di vita e di lavoro che ho raccolto durante i periodi di ricerca sul campo vadano proprio in questa direzione, in questa sede ho scelto di prendere in considerazione solamente alcuni tratti della vita quotidiana di uno dei miei interlocutori privilegiati: Badara Ngom.

Badara Ngom $^{12}$ ha poco più di trent'anni ed è arrivato alla discarica di Mbeubeuss più di dieci anni fa. Nato nella città di Touba (regione di Diourbel) e di origine serere, aveva raggiunto il fratello maggiore a Dakar nella speranza di trovare un lavoro stabile nella capitale. Mi raccontò di essere arrivato quasi per caso a Mbeubeuss per il fatto che, come accade a molti, conosceva dei suoi concittadini impiegati come recuperatori informali nella discarica. Badara ha iniziato come recuperatore e, negli anni, è riuscito a migliorare la sua condizione lavorativa: al momento egli compra plastica dai recuperatori e dai grossisti (boroom-pàkk) per rivenderla a una delle tre fabbriche di proprietà cinese che, da qualche anno, sono state edificate al confine sud-ovest e al confine nord-est della discarica. Anche se i cambiamenti nelle traiettorie di vita delle persone non sono così rapidi come quelli dei luoghi che costituiscono la discarica, essi hanno un notevole impatto sulle aspettative per il futuro e sulla quotidianità. Da qualche anno a questa parte, Badara è riuscito ad affittare la stanza di una grande casa situata accanto al terminal degli autobus di Keur Massar e ciò gli permette di evitare di trascorrere la notte nella discarica. Attualmente, il suo lavoro inizia la mattina presto e termina al calare del sole. Dopo aver fatto colazione presso la baracca di una ristoratrice accanto all'ingresso principale della discarica, raggiunge una tenda posta accanto all'ingresso dell'unità industriale cinese per cui lavora. Lì indossa la sua tunica da lavoro e prende un grosso bastone di legno cui è attaccata una piccola bilancia di colore azzurro e di forma rotondeggiante. Badara porta sempre con sé anche una borsa a tracolla in cui conserva un quaderno, una penna, una calcolatrice e una discreta quantità di denaro in monete o banconote di piccolo taglio (500, 1000, $2000 \mathrm{XFO})$. Spostandosi tra i pàkk di Gouye-gui e di Baol portando il bastone in spalla, Badara urla il nome dei materiali che vuole acquistare: polietilene (PE), polipropilene (PP) o polietilentereftalato (PET). Quando Badara viene chiamato da qualche venditore, suo compito è quello di pesare la plastica - precedentemente raccolta all'interno di una zanzariera - agganciando la bilancia alla zanzariera e, coinvolgendo il venditore, portando il bastone in orizzontale sopra le rispettive teste. Dopo aver segnato il peso sulla pagina del quaderno relativa alla giornata, Badara calcola il prezzo e paga il venditore. La zanzariera di plastica viene lasciata al venditore. Solo una volta raggiunta una certa quantità ( 2 o 3 tonnellate), Badara potrà tornare a prendere le zanzariere da lui acquistate per portarle, a bordo di un camion, alla fabbrica cinese. Lì potrà rivenderle a poco più del doppio del prezzo di acquisto. Badara non sa di preciso che cosa avvenga alla plastica che vende ai cinesi; sa per certo, tuttavia, che una volta trasformata all'interno dei capannoni, viene trasferita al porto e imbarcata alla volta della Cina. La plastica recuperata e venduta riacquista valore di scambio, tuttavia il suo valore d'uso resta celato negli anelli delle catene del mercato transnazionale ${ }^{13}$. Molti altri oggetti, invece, seguono percorsi più facilmente tracciabili: gli zaini, dopo essere stati ricuciti in appositi pàkk, vengono rivenduti nei mercati locali, così come gli abiti; i resti di cibo vengono rivenduti come mangime per gli animali allevati nella discarica e nelle zone limitrofe. I brevi cenni relativi ad alcune delle tipologie di oggetti presente nella discarica mostrano come i rifiuti siano co-costruttori di relazioni sociali, 
economiche e lavorative e che sui rifiuti si reggano tanto economie circolari quanto capitaliste. La traiettoria della plastica passa da una discarica locale al mercato transnazionale e il suo valore di scambio aumenta durante le varie fasi di recupero e compravendita. Le storie di zaini, abiti e scarpe gettano luce su una serie di network economici locali, variabili in base alla tipologia di rifiuto e alla quantità.

La complessa e non sempre efficace gestione locale e statale dei rifiuti ha permesso a centinaia di uomini e donne di guadagnare una vita attraverso un bricolage sociale ed economico. Non tutti i recuperatori hanno scelto di lavorare in discarica: solamente per alcuni la loro attività è considerata il frutto di eventi sfortunati e vicissitudini della vita (la morte prematura di uno o di entrambi i genitori, il divorzio, l'impossibilità di trovare un lavoro velocemente). Molti migranti interni al Paese sostengono che la loro attività è tenuta nascosta alle famiglie di origine. Il sentimento di vergogna e il senso di fallimento permea le storie di alcuni recuperatori. Per altri, invece, la situazione è completamente diversa: è possibile trovare anche coppie di marito e moglie che lavorano nella discarica - in molti casi con ruoli diversi. In ogni caso, tutti i miei interlocutori non vorrebbero vedere i propri figli lavorare nella discarica. Interrogato su quali fossero i suoi desideri per il futuro, Badara non ha mai mostrato imbarazzo nell'affermare di voler continuare a lavorare a Mbeubeuss tuttavia, come molti suoi colleghi, lamentava il fatto che l'assenza di un riconoscimento formale del proprio lavoro rendesse precaria la sua vita lavorativa e, per questo, caricasse di imprevedibili responsabilità nei confronti della sua famiglia. Per Badara, come per molti altri lavoratori della discarica, i rifiuti hanno un profondo significato economico e sociale.

Nella loro dimensione di oggetti - che porta con sé la materialità - i rifiuti consentono a Badara di guadagnare il denaro sufficiente per mantenere la propria famiglia che, dopo la recente scomparsa di sua madre e di suo fratello maggiore (estate 2017), comprende sua moglie, i suoi tre figli, suo fratello e sua sorella - entrambi minori di lui. Inoltre, nella loro materialità, i rifiuti rappresentano anche una fonte di pericolo sia diretto sia indiretto: come detto, i rischi del lavoro in discarica (non soltanto del recupero) sono legati al contatto diretto e alla possibilità di contaminazione (sia dei corpi sia dell'ambiente) visibile e invisibile. Numerosi sono anche gli incidenti che coinvolgono persone, camion e carretti costretti a percorrere strade strette e, spesso, piene di rifiuti di metallo lasciati lì per essere schiacciati dai mezzi pesanti di passaggio. Anche sul corpo di Badara si leggono i segni del pericolo rappresentato dai rifiuti. «Guarda qui mi disse un giorno alzandosi la manica della sua tunica da lavoro e indicandomi una cicatrice sul suo braccio - questa me la sono fatta una notte con un televisore qui in discarica».

\section{Conclusioni}

In questo articolo ho preso in esame la complessa realtà dei rifiuti, partendo dalle categorie e da alcuni esempi tratti dalla letteratura e dalla filosofia classica e arrivando a descrivere alcuni tratti della traiettoria di vita e di lavoro di uno dei recuperatori della discarica di Mbeubeuss, interlocutore privilegiato per la ricerca in Senegal. Dal punto di vista teorico, i rifiuti non risultano essere entità in grado di gettare luce su dinamiche sociali di per sé, come se fossero tracce o testimonianze che attendono di essere scoperte e interpretate. Inoltre, non sono tanto i rifiuti di per se stessi ad avere un valore: come sostiene Reno per la discarica di Four Corners, infatti, esso è dato dai 
contratti che vengono firmati per la loro gestione e lo smaltimento. A Mbeubeuss, invece, il valore del rifiuto può essere legato alla sua materialità, alle sue condizioni e, in base al materiale, alla sua qualità o alla sua quantità - nei casi, ad esempio, dei capi di abbigliamento o della plastica. Se da un primo livello di analisi, pertanto, l'indicazione del valore di scambio di una materia recuperata sembra travalicare la legge domanda-offerta, in realtà è ancora una volta il libero mercato informale dominato da quello formale - a stabilire i prezzi. Sono infatti i proprietari delle aziende cinesi a imporre un prezzo di acquisto della plastica ai singoli grossisti o semi-grossisti che, a loro volta, imporrano un prezzo al ribasso ai recuperatori per l'acquisto del prodotto recuperato. In questo senso, possiamo considerare i recuperatori di rifiuti di Mbeubeuss come intermediari tra istanze del mercato globale ed esigenze individuale di sostentamento e la presenza dei rifiuti come la condizione necessaria per la materializzazione di queste istanze. In conclusione, l'analisi che ho presentato in queste pagine tiene insieme le dimensioni multi-scalari delle relazioni tra individui e rifiuti attraverso il filtro dello spazio (Soja 2010) e pone come limiti da una parte i corpi dei miei interlocutori e delle mie interlocutrici e, dall'altra, i confini degli spazi di riflessione teorica.

\section{BIBLIOGRAFIA}

Alexander C., O'Hare P., 2020 «Waste and Its Disguises: Technologies of (Un)Knowing», in Ethnos, DOI: $10.1080 / 00141844.2020 .1796734$

Alliegro E.V., 2017 «Simboli e processi di costruzione simbolica. La “Terra dei Fuochi” in Campania», in EtnoAntropologia, 5, 2: 175-239.

Alliegro E.V., 2018 «Rimasugli, scarti, rifiuti tra waste anthropology e Terra dei Fuochi», in Voci, $\mathrm{XV}: 137-165$.

Bauman Z., 2007 Vite di scarto, Laterza, Roma-Bari.

Calvino I., 1994 Romanzi e racconti, Mondadori, Milano, Volume 3.

Calvino I., 2016 [1972] Le città invisibili, Mondadori, Milano.

Clemente P., Rossi E., (a cura di), 1999 Il terzo principio della museografia. Antropologia, contadini, musei, Carocci, Roma.

Cissé O., 2007 L'argent des déchets : L'économie informelle à Dakar, Karthala, Dakar-Parigi.

Cissé O. (éd.), 2012 Les décharges d'ordures en Afrique. Mbeubeuss à Dakar au Sénégal, IAGU-Karthala, Dakar-Parigi.

Diawara A.B., 2010 Les déchets solides a Dakar. Environnement, sociétés et gestion urbaine, Tesi di dottorato in Geografia, Université Michel de Montaigne, Bordeaux III, Bordeaux https:// tel.archives-ouvertes.fr/tel-00466516 (ultimo accesso 6 giugno 2020).

Douglas M., [1966] 2014 Purezza e pericolo. Un'analisi dei concetti di contaminazione e tabù, Il Mulino, Bologna. 
Drackner M., 2005 «What is waste? To whom? - An anthropological perspective on garbage», in Waste Management \& Research, 23: 175-181.

Eriksen T.H., 2017 Fuori controllo. Un'antropologia del cambiamento accelerato, Einaudi, Torino.

Fall, A.S., 2007 Bricoler pour survivre. Perceptions de la pauvreté dans l'agglomération urbaine de Dakar, Karthala, Parigi, Dakar.

Fredericks R., 2018 Garbage Citizenship: Vital Infrastructures of Labor in Dakar, Senegal, Duke University Press Durham and London.

Kopytoff I., 1986 «The Cultural Biography of Things. Commoditization as process», in A. Appadurai (ed.) The Social Life of Things. Commodities in Cultural Perspective, Cambridge University Press: 64-91.

Meloni P., 2011 I modi giusti. Cultura materiale e pratiche di consumo nella provincia toscana, Pacini, Pisa.

Melosi M.V., 2005 Garbage in the Cities. Refuse, Reform, and the Environment, University of Pittsburgh Press, Pittsburgh

Millar K.M., 2018 Reclaiming the Discarded. Life and Labor on Rio's Garbage Dump, Duke University Press, Durham e Londra.

Nguyen M.T.N., 2019 Waste and Wealth. An Ethnography of Labor, Value, and Morality in Vietnamese Recycling Economy, Oxford University Press.

O’Brien M., 2008 Understanding the Rubbish Society, Routledge, London.

Rathje W., Murphy, C. ,1992 Rubbish! The Archaeology of Garbage, Harper Collins, New York.

Reale G., 2000 Platone. Tutte le opere. Bompiani, Milano: 379-386.

Reno J.O., 2014 «Toward a new theory of waste: from "matter out of place" to "signs of life"», in Theory, Culture and Society, XXXI: 3-27.

Reno J.O., 2016 Waste away: Working and Living with a North American Landfill, University of California Press, Berkeley.

Rimoldi L., 2018 «Un disastro di lunga durata. Pratiche di gestione dei rifiuti nel Senegal contemporaneo», in Illuminazioni, 8, 6: 29-63.

Rimoldi L., 2019a «Spazi marginali e rifiuti in Senegal. Riflessioni etnografiche sulla discarica di Mbeubeuss (Dakar)», in Tracce Urbane, 5: 110-123.

Rimoldi L., 2019b « Badara Ngom et la décharge. Une réflexion anthropologique sur les déchets de Mbeubeuss, Senegal », in Trois/Cent/Soixante, 3, Marchandise, http://bit.ly/2VzELeg.

Scarpino C., 2011 US Waste. Rifiuti e sprechi d'America: una storia dal basso, Il Saggiatore, Milano.

Soja E.W., 2010 Seeking Spatial Justice, University of Minnesota Press, Minneapolis-Londra.

Thill B., 2015 Waste, Bloomsbury, New York.

Thompson M., 2017 [1979] Rubbish Theory: The Creation and Destruction of Value, Pluto Press, Londra.

Valéry P., 2011 Eupalinos o l'Architetto, Mimesis, Milano-Udine.

Viale G., 1994 Un mondo usa e getta. La civiltà dei rifiuti e i rifiuti della civiltà, Feltrinelli, Milano. 


\section{NOTE}

1. I dati etnografici presentati in questo articolo sono stati raccolti grazie a numerosi periodi di ricerca sul campo effettuati tra il 2016 e il 2019 trascorsi insieme ai lavoratori (formali e informali) di Mbeubeuss, durante $i$ quali ho sia realizzato interviste semi-strutturate sia partecipato attivamente al lavoro quotidiano di raccolta, prima, e di compravendita dei rifiuti, in una fase successiva. La ricerca è stata finanziata da un assegno di ricerca (A2 Junior) del Dipartimento di Sociologia e Ricerca Sociale dell'Università degli Studi di Milano-Bicocca, da un contratto di ricerca con SWAB - Shadows of Slavery in West Africa and Beyond: A Historical Anthropology - ERC grant agreement $n^{\circ} 313737$, cofinanziata dalla Missione Etnologica in Benin e Africa Occidentale (MEBAO) e dalla Missione Etnologica in Senegal e Africa Occidentale (MESAO) del Ministero degli Affari Esteri.

2. Joshua Reno ha condotto una ricerca etnografica in nord America tra il 2004 e il 2007 e ha coinvolto lavoratori dei rifiuti e attivisti attraverso interviste qualitative e raccolta di storie di vita e di lavoro. Oltre a ciò, Reno ha lavorato per nove mesi all'interno di una grande discarica dello Stato del Michigan che chiama "Four Corners" come recuperatore di carta, con l'obiettivo dichiarato di comprendere in profondità le influenze dei rifiuti sulle vite e sui corpi dei lavoratori della discarica, ricercatore compreso.

3. I recuperatori e le recuperatrici di Mbeubeuss vengono chiamati - e si chiamano tra loro nelle interazioni scherzose quotidiane - boudioumane. Il termine spregiativo indica, in lingua wolof, proprio il rovistare a terra tra i rifiuti in cerca di qualcosa.

4. Il racconto fu pubblicato per la prima volta nel 1977 in "Paragone/Letteratura" e, successivamente, nel postumo La strada di San Giovanni (Bologna 2003: 54). I riferimenti nel testo sono, invece, alla versione pubblicata da Mondadori nel 1994 in Romanzi e Racconti (Volume terzo), edizione diretta da Claudio Milanini e curata da Mario Barenghi e Bruno Falcetto.

5. I rifiuti recuperati con finalità artistico-trasformative sono da tempo oggetto di analisi da parte dell'antropologia italiana. Per approfondire queste tematiche si vedano, ad esempio Clemente 1999: 23-41 e Lusini 2015.

6. A questo proposito si veda anche Meloni 2011.

7. Il riferimento è alla prima parte del Parmenide (128E-135C) tradotto, presentato e annotato da Maurizio Migliori (pp. 379-386) in Platone. Tutte le opere, a cura di Giovanni Reale (2000) edito da Bompiani.

8. Tale immaginario legittima i discorsi sulle marginalità che, nella retorica delle istituzioni locali, nazionali e internazionali, caratterizza le vite dei boudioumane di Mbeubeuss. Per approfondire si vedano Rimoldi 2018 e Rimoldi 2019a, 2019 b.

9. Nei primi decenni dopo l'Indipendenza del Senegal, la raccolta dei rifiuti a Dakar era gestita dai singoli Comuni a cui era affidato il trasporto nelle due discariche presenti sul territorio urbano. Tra l'inizio degli anni settanta e la metà degli anni ottanta fu una società privata - la Société africaine de diffusion et de promotion (SOADIP) - ad occuparsi dei rifiuti della capitale. Tra la metà degli anni ottanta e la metà degli anni novanta si avvicendarono la Communauté urbaine de Dakar (CUD) e la Société industrielle d'aménagement du Sénégal (SIAS); fu poi la volta della CUD-AGETIP che gestì i rifiuti urbani cittadini fino a quando, all'inizio degli anni Duemila, l'allora presidente Wade creò l'Agence pour la propreté de Dakar (APRODAK) a cui succedette l'ALYCON-AMA Sénégal (2002). Tra il 2006 e il 2011 la gestione dei rifiuti venne affidata alla CADAK-CAR, sotto il controllo del Comune di Dakar e di quello di Rufisque e, solo nel 2015, lo Stato tornò ad occuparsi della gestione dei rifiuti attraverso l'UGC (Cfr. Cissé 2007: 21-48, Diawara 2009, Fredericks 2018).

10. Le relazioni tra i lavoratori dei diversi insediamenti non sono sempre pacifiche. Ad esempio, $i$ Baol-Baol spesso accusano i lavoratori di Gouye-gui di fare accordi illegali con gli autisti dei camion per fare in modo che questi ultimi depositino i rifiuti trasportati direttamente nei pàkk di 
Gouye-gui e non sulle piattaforme. Questo non solo crea tensione tra i lavoratori informali, ma costringe i lavoratori dell'UCG ad effettuare controlli più rigorosi sui percorsi dei camion. I conflitti tra i vari insediamenti sono in parte risolti attraverso i rappresentanti delle zone eletti all'interno dell'Associazione dei recuperatori e delle recuperatrici di Mbeubeuss (Bokk-Diom).

11. I carretti e i loro proprietari sono nella maggior parte dei casi originari delle regioni agricole del Senegal e, come anche alcuni recuperatori, si occupano del trasporto dei rifiuti da un luogo a un altro della discarica solamente quando non impegnati nei lavori agricoli. Le corse sui carretti costano pochi spiccioli, ma sono fondamentali per il mantenimento dell'economia della discarica di Mbeubeuss.

12. Il nome utilizzato è di fantasia ed è stato scelto dal diretto interessato.

13. Sulle biografie degli oggetti si veda Kopytoff 1986.

\section{RIASSUNTI}

Questo contributo descrive alcuni tratti della vita sociale dei rifiuti, inserendosi nel dibattito sull'antropologia della loro vita materiale e sociale, per poi concentrarsi sugli spazi di vita e di lavoro della discarica di Mbeubeuss, nella periferia di Dakar. I rifiuti sono in grado di creare relazioni, spazi e paesaggi delimitati e definiti dalle politiche e dalle pratiche (formali e informali) della loro stessa gestione. La riflessione qui presentata poggia le sue basi su parte dei dati etnografici raccolti tra il 2016 e il 2019 presso un gruppo di lavoratrici e lavoratori formali e informali della discarica, e si focalizza su alcuni eventi della vita quotidiana di uno di loro Badara Ngom. Obiettivo di questo lavoro è analizzare le modalità attraverso le quali i rifiuti costruiscono spazi sociali e relazionali e dimostrare come il valore non sia solamente una rappresentazione delle relazioni sociali, ma contribuisce a orientare traiettorie di vita e mantenere un ordine sociale legato ai rifiuti.

This article describes some aspects of the social life of waste, contributing to the debate on anthropology of waste, and focusing on the living and working spaces of the Mbeubeuss landfill, on the outskirts of Dakar. From a theoretical point of view, waste escapes a rigid categorization, yet they create relationships, spaces and landscapes delimited and defined by the policies and (formal and informal) practices of their own management. The reflection presented here, based on ethnographic data collected between 2016 and 2019 with a group of formal and informal workers of Mbeubeuss, aims to analyze the construction of social and relational spaces in the Dakar landfill. I intend to demonstrate how value is not only a representation of social relations, but how it contributes to orienting life trajectories, and to maintaining a social order.

\section{INDICE}

Parole chiave : antropologia, etnografia, rifiuti, spazio, Dakar

Keywords : anthropology, ethnography, waste, space, Dakar 


\section{AUTORE}

\section{LUCA RIMOLDI}

Dipartimento di Scienze Politiche e Sociali, Università degli Studi di Catania luca.rimoldi@unict.it 